\title{
Angiopoietin-1/Tie-2 activation contributes to vascular survival and tumor growth during VEGF blockade
}

\author{
JIANZHONG HUANG ${ }^{1}$, JAE-O BAE ${ }^{1}$, JUDY P. TSAI ${ }^{1}$, ANGELA KADENHE-CHIWESHE ${ }^{1}$, JOEY PAPA ${ }^{1}$, \\ ALICE LEE $^{2}$, SHAN ZENG ${ }^{1}$, Z. NOAH KORNFELD ${ }^{1}$, PAIVI ULLNER ${ }^{1}$, NIBAL ZAGHLOUL ${ }^{2}$, ELLA IOFFE ${ }^{4}$, \\ SARAH NANDOR ${ }^{4}$, ELENA BUROVA ${ }^{4}$, JOCELYN HOLASH $^{4}$, GAVIN THURSTON $^{4}$, JOHN RUDGE $^{4}$, \\ GEORGE D. YANCOPOULOS ${ }^{4}$, DARRELL J. YAMASHIRO ${ }^{1-3^{*}}$ and JESSICA J. KANDEL ${ }^{1 *}$
}

Departments of ${ }^{1}$ Surgery, ${ }^{2}$ Pediatrics, and ${ }^{3}$ Pathology, College of Physicians and Surgeons of Columbia University, 630 West 168th Street, New York, NY 10032; ${ }^{4}$ Regeneron Pharmaceuticals,

777 Old Saw Mill River Road, Tarrytown, NY 10591, USA

Received July 11, 2008; Accepted September 19, 2008

DOI: 10.3892/ijo_00000131

\begin{abstract}
Approval of the anti-vascular endothelial growth factor (VEGF) antibody bevacizumab by the FDA in 2004 reflected the success of this vascular targeting strategy in extending survival in patients with advanced cancers. However, consistent with previous reports that experimental tumors can grow or recur during VEGF blockade, it has become clear that many patients treated with VEGF inhibitors will ultimately develop progressive disease. Previous studies have shown that disruption of VEGF signaling in tumors induces remodeling in surviving vessels, and link increased expression of angiopoietin-1 (Ang-1) with this process. However, overexpression of Ang-1 in different tumors has yielded divergent results, restricting angiogenesis in some systems while promoting it in others. These data raise the possibility that effects of Ang-1/Tie-2 may be context-dependent. Expression of an Ang-1 construct (Ang1*) did not significantly change tumor growth in our model prior to treatment, although vessels exhibited changes consistent with increased Tie-2 signaling. During inhibition of VEGF, however, both over-
\end{abstract}

Correspondence to: Dr Jessica J. Kandel, Pediatric Surgery, Children's Hospital 214N, 3959 Broadway, New York, NY 10032, USA

E-mail: jjk47@columbia.edu

*Both senior authors contributed equally

Abbreviations: $\alpha$ SMA, $\alpha$-smooth muscle actin; Ang-1, angiopoietin-1; PDGF-B, platelet-derived growth factor B; PECAM-1, platelet-endothelial cell adhesion molecule-1; VEGF, vascular endothelial growth factor

Key words: angiogenesis, tumor growth, angiopoietin-1, Tie-2, VEGF, vascular remodeling expression of Ang $1^{*}$ and administration of an engineered Ang-1 agonist (Bow-Ang1) strikingly protected tumors and vasculature from regression. In this context, Ang-1/Tie-2 activation limited tumor hypoxia, increased vessel caliber, and promoted recruitment of mural cells. Thus, these studies support a model in which activation of Tie-2 is important for tumor and vessel survival when VEGF-dependent vasculature is stressed. Understanding such mechanisms of adaptation to this validated form of therapy may be important in designing regimens that make the best use of this approach.

\section{Introduction}

The development of a variety of agents which bind vascular endothelial growth factor (VEGF) or block its signaling via VEGF receptors (VEGFRs) has resulted in a body of research demonstrating that interruption of this axis is broadly effective in inhibiting pathologic angiogenesis. The accumulating clinical experience with the anti-VEGF antibody bevacizumab has largely borne out the initial experimental findings $(1,2)$. Yet these results have equally clearly presented a new problem: virtually all patients who receive this form of therapy will ultimately progress. These observations raise the question of whether alternative proangiogenic mechanisms can support tumor vessels when VEGF signaling is blocked.

Withdrawal of VEGF can cause small endothelial vessels lacking pericytes to undergo rapid apoptosis, while those with associated mural cells preferentially survive $(3,4)$. Vasculature that persists during VEGF blockade demonstrates mural cell recruitment and perivascular expression of molecules implicated in vascular remodeling, including angiopoietin-1 (Ang-1) (5-8). Yet the role of Ang-1 signaling in tumorigenesis has been difficult to define, with widely varying results of overexpressing this factor in different tumor systems. Some workers have found significant restriction of tumor growth and angiogenesis in models of experimental colon cancer and squamous cell carcinoma $(9,10)$. Conversely, Machein et al and Shim and coworkers noted increased tumor growth in glioma and cervical cancer models $(11,12)$. 
One potential explanation for these differences may be that the effects of Ang-1 are context-dependent. For example, Tie-2 activation may enhance endothelial survival signaling and tumor perfusion in conditions of microenvironmental stress, when other signals are relatively deficient (13-15). In support of this concept, Winkler and coworkers have reported that VEGF inhibition can induce expression of Ang-1, which is associated with improved tumor perfusion (16). Casanovas et al have reported that tumors progressing during treatment with anti-VEGF receptor-2 antibody are not hypoxic, and also noted expression of Ang-1 (7). VEGF can promote proteolytic processing and shedding of the extracellular domain of Tie-2, suggesting that loss of VEGF might increase availability of this receptor (17). Collectively, these observations raised the possibility that Ang-1/Tie-2 activation may function to support tumor vasculature in the specific context of VEGF blockade.

To study this question, we investigated the effect of Ang-1 stimulation in the SK-NEP1 model, which we have previously shown to be highly susceptible to destabilization by VEGF blockade (18). In these studies we find that overexpression of an Ang-1 construct (Ang1*) neither limited nor promoted initial growth of tumors prior to VEGF Trap treatment. Instead, stimulation of this axis by Ang1* remodeled vasculature. Overexpression of Ang $1 *$ or administration of the engineered tetrameric Tie-2 agonist BowAng1 (19) functioned to stabilize vasculature, rendering xenografts resistant to regression during blockade of VEGF. When VEGF was inhibited, vascular remodeling and survival signaling increased in Ang1* tumors as compared to controls, whereas hypoxia was reduced. Thus, these results indicate that Ang-1/Tie-2 function exerts specific effects on tumor vasculature that are functionally important in the context of VEGF inhibition, supporting the potential significance of this pathway as a preconditioning or compensatory mechanism affecting the response to anti-VEGF treatment.

\section{Materials and methods}

Transfection of SK-NEP-1 cells with the Angl* construct. Cultured human SK-NEP-1 cells (American Type Culture Collection, Manassas, VA) were maintained in McCoy's 5A medium (Mediatech, Fisher Scientific, Springfield, NJ) with $15 \%$ fetal bovine serum and $1 \%$ penicillin-streptomycin (Gibco, Grand Island, NY). Cells were grown at $37^{\circ} \mathrm{C}$ in $5 \%$ $\mathrm{CO}_{2}$ until confluent. The Ang1* construct has been previous described, and retains the agonistic properties of human Ang-1 for Tie-2 (20). Briefly, in Ang1* the nonconserved cysteine at residue 245 of human Ang- 1 has been mutated to the corresponding serine residue of Ang-2, and the first 77 amino acids replaced with the first 73 residues of Ang- 2 . This construct was inserted into a retroviral vector (pLTR) which contains an IRES GFP. Cultured SKNEP1 cells were infected with retrovirus, and sorted by flow cytometry. ELISA analysis and Western blotting of cell culture media were used to verify target protein production. SK-NEP1 cells transduced with the empty vector (SK-NEP1-GFP) were used as controls. To confirm stable expression of the construct throughout the experiment, we first assessed expression of Ang $1^{*}$ by Western blot analysis using anti-human Ang-2
(N-18) antibody (Santa Cruz: sc-7016, 1:500). Total proteins were extracted from the tumor tissues and the cultured cells using cell lysis buffer $(10 \mathrm{mM}$ Tris- $\mathrm{HCl}, \mathrm{pH} 7.4,150 \mathrm{mM}$ $\mathrm{NaCl}, 2$ mM EDTA, 1\% Triton X-100, 10\% glycerol, 2 mM sodium orthovanadate, and complete protease inhibitor cocktail). Equal amounts of proteins were subjected to SDS-PAGE and transferred to nitrocellulose membranes. Proteins were visualized with an enhancer chemiluminescence (ECL) detection kit (Amersham Biosciences, Piscataway, NJ). Erk protein expression detected by stripped same membrane using p44/42 MAP Kinase antibody (Cell Signaling) was used as a loading control.

To confirm that this signal derived from the construct, we combined immunoprecipitation for a $\mathrm{C}$-terminal sequence of Ang-1 with Western blotting for the $\mathrm{N}$-terminal sequence of Ang-2. Total proteins were prepared from the tumor tissues and cultured cells using cell lysis buffer (see above). Equal amounts of proteins were incubated with $0.5 \mu \mathrm{g}$ of primary antibody (anti-human Ang-1, cat no. A 1104, Sigma-Aldrich Corp., St. Louis, MO) and $20 \mu \mathrm{l}$ protein A agarose beads (Santa Cruz) overnight at $4^{\circ} \mathrm{C}$. Samples were centrifuged and the supernatants were discarded. The beads were washed three times with cell lysis buffer and resuspended in $2 \mathrm{X}$ sample buffer. The samples were boiled and subjected to SDS-PAGE and transferred to nitrocellulose membranes, and immunoblotted for Ang-2 as above. A recombinant human Ang-1 protein (R\&D Systems, Inc.) was used as a positive control. We also performed the reverse immunoprecipitation/Western blotting using the same antibodies in reverse order, with an essentially identical result. Last, we performed RT-PCR to confirm construct expression using following primers designed to flank the site where the N-terminal Ang-2 sequence links with Ang-1: 5'-GCA GCC TAT AAC AAC TTT CGG AAG-3'; 5'-GGG TCT CAA CAT CTG TCA GCT TTC-3' (data not shown).

Tie2 phosphorylation. Confluent mouse endothelial cells, bEND (ATCC CRL-2299, Manassas, VA) and human umbilical vein endothelial cells (HUVECs) were starved in plain DMEM medium for $10 \mathrm{~h}$ before addition of recombinant human Ang-1 (R\&D 923-AN), Ang1* or SK-GFP cell culture medium. The cell lysate proteins were immunoprecipitated with anti-Tie2 antibody (Upstate 05-584, Lake Placid, NY) and then Western blotted with anti-phosphotyrosine antibody (Millipore 05-1050). The membrane was stripped and reblotted with anti-Tie2 antibody (R\&D System) to verify equivalent loading of Tie 2 protein.

Tumor model. All experiments were approved by the Institutional Animal Care and Use Committees of either Columbia University (Ang1* experiments) or Regeneron Pharmaceuticals Inc. (BowAng-1 experiments). 4-6-week-old female NCR nude mice (National Cancer Institute-Frederick Cancer Research Center, Frederick, MD) were acclimated to 12-h light-dark cycles in a barrier facility. The left flank was prepared sterilely after anesthesia with intraperitoneal ketamine $(50 \mathrm{mg} / \mathrm{kg})$ and xylazine $(5 \mathrm{mg} / \mathrm{kg})$. The left kidney was exposed via a flank incision and $10^{6}$ tumor cells injected with a 25-gauge needle. After 6 weeks, tumors were palpable in all mice. Animals were randomly divided into two groups 
and injected intraperitoneally twice weekly with VEGF Trap or an equal amount of human Fc protein (both $25 \mathrm{mg} / \mathrm{kg}$; Regeneron Pharmaceuticals, Tarrytown, NY). Animals were sacrificed at day 36 .

BowAng-1 experiments. Tumor implantation was performed as above, and animals with palpable tumors randomly divided into 3 groups after 29 days of growth. Mice were: i) injected subcutaneously with BowAng-1, an engineered tetrameric construct that induces phosphorylation of Tie-2 in cultured endothelial cells (19) (25 mg/kg three times per week); ii) injected subcutaneously with human Fc ( $25 \mathrm{mg} / \mathrm{kg}$ biweekly); or iii) left untreated. These non-treated tumor-bearing mice were euthanized 35 days after implantation to provide control tumor weights and vasculature. At the same time, BowAng-1 pre-treated mice started to receive VEGF Trap $(25 \mathrm{mg} / \mathrm{kg}$ biweekly) and the Fc pre-treated group was randomly subdivided to receive continued Fc injections or VEGF Trap (25 mg/kg biweekly). All tumors were monitored by caliper measurement throughout the course of the study. Mice from each group were euthanized on treatment day 0 , treatment day 5, or treatment day 27 ( $\mathrm{N}=4$ for each group at each point). Before dissection, selected mice were perfused with $4 \%$ paraformaldehyde solution in PBS. Tumors were post-fixed with $4 \%$ paraformaldehyde solution in PBS.

Lectin perfusion. Before death, selected mice at each time-point underwent intravascular injection of fluorescein-labeled Lycopersicon esculentum lectin (100 $\mu \mathrm{g}$ in $100 \mu \mathrm{l}$ saline, Vector Laboratories) into the left ventricle. Vasculature was fixed by infusion of $1 \%$ paraformaldehyde followed by PBS.

Immunohistochemistry. Thick sections $(5-\mu \mathrm{m})$ were deparaffinized in xylene, rehydrated, and endogenous peroxidases quenched in $3 \%$ hydrogen peroxide (Sigma-Aldrich). Specimens were incubated with antibody (PECAM, rat anti-mouse platelet endothelial cell adhesion molecule-1 monoclonal antibody, Research Diagnostics, Inc., Flanders, NJ, 1:50 dilution; $\alpha$ SMA, monoclonal anti- $\alpha$ smooth muscle actin; collagen type IV, CosmoBio, Japan, 1:1000; NG2, Chemicon, CA, 1:100), and biotinylated secondary antibody (Zymed, Grand Island, NY). Enhanced horseradish peroxidaseconjugated streptavidin and a substrate chromogen, AEC (3-amino-9-ethyl carbazole), were used to visualize signals. Immunofluorescence was performed on frozen specimens. Sections $(5-\mu \mathrm{m})$ were cut from tumors embedded in OCT and stored at $-80^{\circ} \mathrm{C}$. Slides were brought to room temperature, washed in ice-cold acetone for $10 \mathrm{~min}$, incubated with avidin/ biotin, CAS blocking solutions and primary antibody as described above. A biotinylated secondary antibody was used in combination with fluorescein-labeled avidin to visualize signals. Slides were examined with a Nikon Eclipse E600 or a Zeiss Axiophot microscope. Four magnification $x 400$ fields in 4 different tumors were examined.

Quantitation of gene expression by real-time PCR. Total RNA was extracted from tumor tissues using Rneasy Plus Mini Kit (Qiagen) and was processed directly to cDNA synthesis using TaqMan ${ }^{\circledR}$ Reverse Transcription Reagents Kit (Applied Biosystems). PCR primers and TaqMan probes for human
VEGF (Hs00173626_m1) and CXCL12 (Hs00171022_m1) were purchased from Applied Biosystems. Human B-actin (Applied Biosystems) was used as an endogenous reference. Quantitative real-time PCR was performed using the ABI PRISM 7900 Sequence Detection System (Applied Biosystems). Data are calculated by $2-\Delta \Delta \mathrm{Ct}$ method as described by the manufacturer and are expressed as the fold increase over the indicated controls (1.0) in each figure.

Digital image analysis. Digital images from immunofluorescence and fluorescein-labeled lectin studies were captured from a Nikon E600 fluorescence microscope with a Spot RT slider digital camera (Diagnostic Instruments, Sterling Heights, MI) and stored as TIFF files. Vessel radius was determined by immunofluorescence for $\alpha \mathrm{SMA}$ and analyzed using software Adobe Photoshop 7.0 (Adobe Systems, Mountain View, CA) and Image Processing Toolkit (IPTK 5 IPTK 5, Reindeer Graphics, Raleigh, NC). Briefly, background fluorescence is subtracted (AutoLevelDark), Gaussian filter applied to reduce electronic and background noise (Gaussian Blur, radius $=1.0$ pixel), and grayscale levels linearly expanded (Auto Levels). A common threshold level is applied that preserves correct vascular morphology (Threshold). The image is inverted (Invert) and dilated to lessen gaps (Classic Morphology $>$ Dilate), small particles representing background removed (Reject Features), and vessel holes filled in (Fill Holes). The average of the inscribed radius is determined as a measure of vessel radius. Sixty images from SK-NEP1-GFP (3 different tumors) and 49 images from SK-NEP1-Ang1* (3 different tumors) were analyzed. To assess MVD and vessel length, quantitative assessment of tumor vessel architecture was performed by computer-assisted digital image analysis as previously described (21). The fraction of fluorescein-labeled lectin-positive pixels per total field was quantified. Specific changes in vessel architecture are evaluated by quantifying branch points (nodes), end-points, and total vessel length. Images are analyzed after application of a common threshold, image inversion, morphological erosion, and skeletonization, again using a combination of Photoshop and Image Processing Toolkit.

Statistical analysis. Statistical comparison was performed using the Analyse-It add-in software package for Microsoft Excel. Kruskal-Wallis analysis was used to compare tumor weights and numeric values resulting from computerized image analysis.

\section{Results}

Ang $1 *$ expression induces vessel remodeling but does not alter tumor size. To examine the role of Ang-1/Tie-2 activation in the setting of VEGF blockade, we utilized the SK-NEP1 model. We have previously shown that SK-NEP1 xenografts are relatively VEGF-dependent, and can be significantly regressed by VEGF Trap (18). SK-NEP1 cells were engineered to express either the Ang1* construct, in which the N-terminal sequence is replaced by that of Ang-2, and which has previously shown to be a potent Tie-2 agonist comparable to native Ang-1 (20), or GFP (a schematic comparison of Ang-1, Ang 1*, and BowAng-1 is presented in Fig. 1A). We confirmed 
A

Ang1

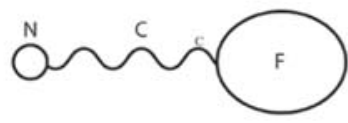

Ang $1^{*}$
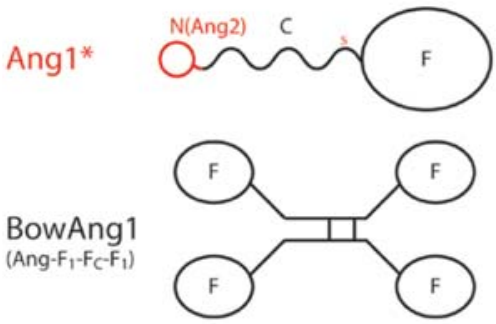

$\mathrm{B}$

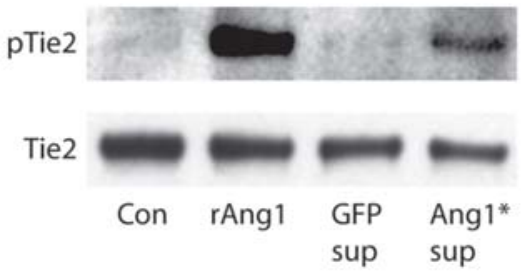

C

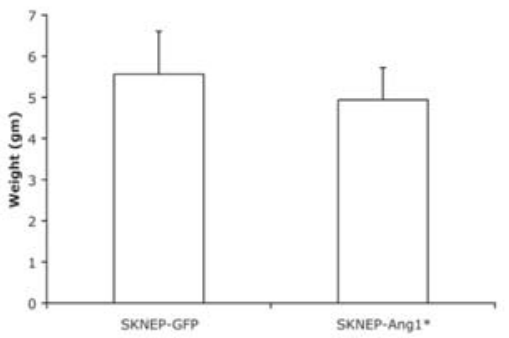

$E$

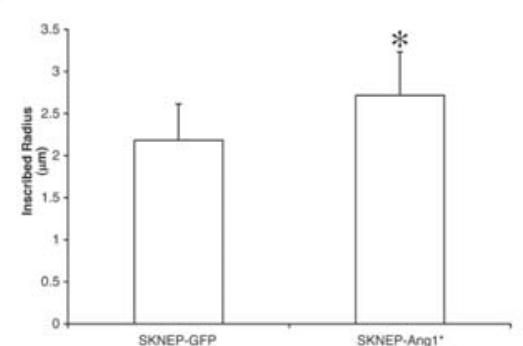

D
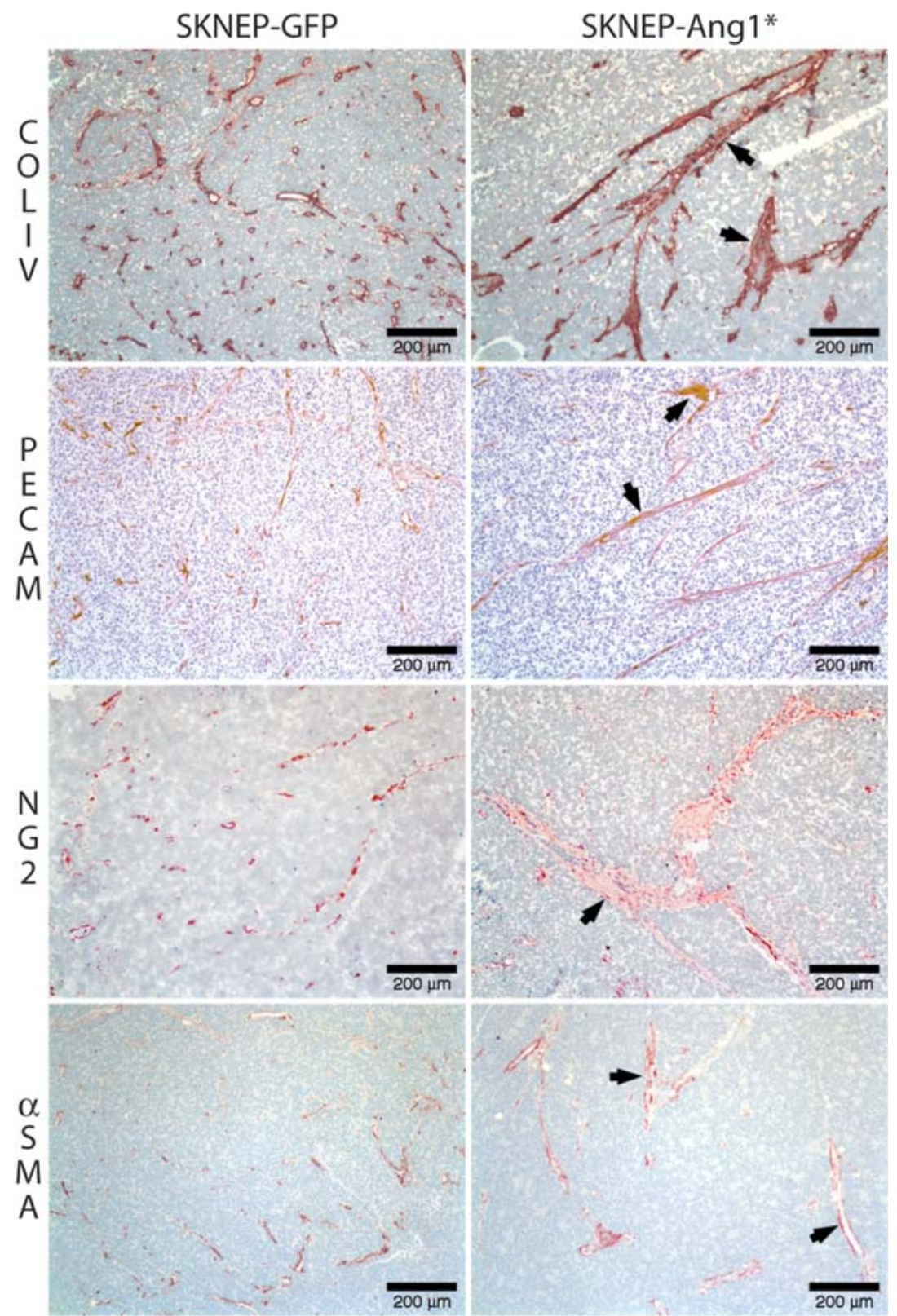

Figure 1. Tumor vasculature is altered by expression of Ang1*. (A) Schematic comparison of native Ang-1, Ang1*, and Bow-Ang1. The Ang1* construct differs from native Ang-1 in that the N-terminal 77 amino acids are replaced by the 73 N-terminal amino acids of Ang- 2 . The ability to activate Tie-2 resides in the C-terminal fibrinogen-like domain, and its bioactivity therefore resembles native Ang-1. BowAng1 is a tetrameric Ang-1 mimetic which takes advantage of the requirement for higher-order multimers for Tie-2 activation, and which potently stimulates this axis (19). (B) Ang $1 *$ secreted by SKNEP-1 cells stimulates Tie-2 phosphorylation in cultured human endothelial cells. Recombinant human Ang-1 was used as a control. Stimulation was less pronounced but also present in murine endothelioma cells (BEnd3). (C) Tumor weights did not differ in Ang1*-and GFP-expressing SK-NEP1 xenografts after the initial 5.5 weeks of growth. (D) Vasculature is remodeled in Ang1* tumors as compared to GFP-expressing controls. Immunostaining for vascular basement membrane (Collagen IV) and endothelial cell (PECAM) markers demonstrates a decrease in fine branching in Ang $1 *$ tumors. There is a distinct increase in recruitment of cells expressing the nascent pericyte marker NG2(+)to vessels, without a corresponding increase in the marker for the more differentiated mural cells (alpha smooth muscle actin, $\alpha \mathrm{SMA}$ ). (E) Corresponding to the greater predominance of large caliber vessels in Ang ${ }^{*}$ tumors suggested by immunostaining, mean vascular radius is increased $124.4 \%$ versus GFP-transfected xenografts at day 0 (P<0.0001).

that Ang1* secreted from our tumor cells could activate Tie-2 in cultured endothelium (Fig. 1B). Ang1*- and control GFP-transfected SK-NEP1 cells were implanted in nude mice, and allowed to grow for 5 weeks. At this point (day 0) a cohort of animals was sacrificed for analysis of vessel architecture and tumor weights. We reasoned that Ang- $1 /$ Tie-2 signaling might alter vascular assembly by promoting remodeling and maturation of vessel networks, potentially influencing tumor perfusion and growth. However, expression of Ang $1 *$ did not change tumor weight as compare to GFP- 


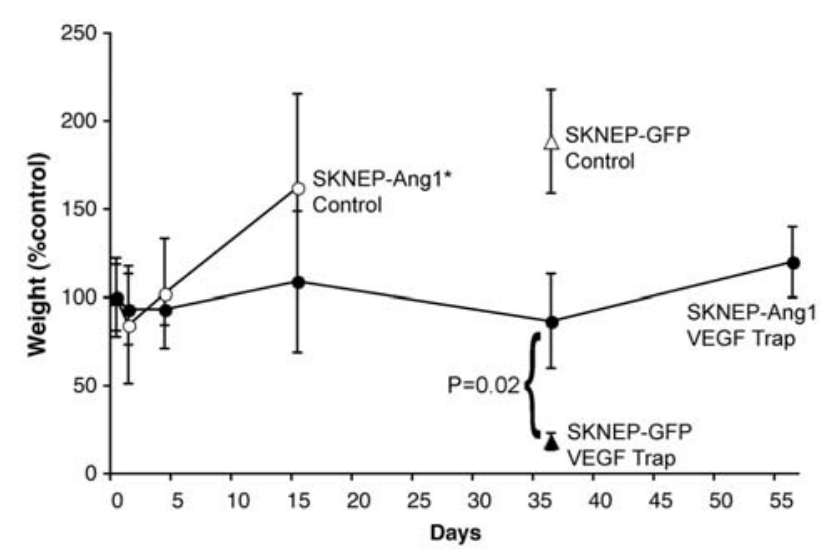

Figure 2. Tumors engineered to over-express an angiopoietin-1 construct are not regressed by VEGF blockade. Xenografts expressing Ang1* or GFP were allowed to grow for 6 weeks, then treated with $25 \mathrm{mg} / \mathrm{kg}$ VEGF Trap biweekly. Whereas GFP-expressing tumors were regressed by $80 \%$ from starting weights by day 36 of treatment with VEGF Trap (filled triangles; $5.6 \pm 1.1 \mathrm{~g}$ at day 0 vs. $1.0 \pm 0.3 \mathrm{~g}$ at day $36, \mathrm{P}=0.0003)$, Ang $1 *$-expressing tumors were not regressed (filled circles). Fc-treated Ang1* controls and GFP controls grew progressively (empty circles, Ang1* Fc-treated; empty triangle, GFP controls Fc-treated).

transfected controls (Fig. 1C). Thus, despite ability of Ang1* to activate Tie-2, increased activity of this axis did not affect tumor growth in our model.

Because Ang-1 may also alter recruitment of different vascular cell types (e.g., Tie-2 expressing endothelial and pericyte progenitors) to forming vessels, we examined these components. Immunostaining for the endothelial marker platelet-endothelial cell adhesion molecule-1 (PECAM) demonstrated decreased branching in Ang1* expressing tumors, consistent with a shift to larger-caliber vasculature (Fig. 1D). To examine changes in recruitment of vascular progenitor cells, we immunostained for neural-glial 2 (NG2), a marker for early pericytes. Intriguingly, the distribution of $\mathrm{NG} 2(+)$ cells differed distinctly in Ang1* tumors, with intraluminal cells present. In contrast, similar distribution of alpha-smooth muscle actin ( $\alpha \mathrm{SMA})$, a marker for differentiated vascular mural cells, was found in both tumor types, although Ang1* tumors were relatively enriched in $\alpha \mathrm{SMA}(+)$ largecaliber vessels (Fig. 1D).

One established effect of Ang-1/Tie-2 activation is to increase the caliber of vessels, as seen in the skin of transgenic mice (22). Therefore, we used computer-assisted image analysis of fluorescent immunostaining for perivascular mural cells ( $\alpha$ SMA) to compare vessel diameters in Ang $1 *_{\text {- }}$ and GFP-expressing tumors at day 0 . The mean inscribed radius of Ang1*-tumor vessels increased to $124 \%$ of GFP-transfected control vasculature $(\mathrm{P}<0.0001$; Fig. 1E). Taken together, these data suggest that SK-NEP1 tumors which express Ang1* recruit vascular cells and form vessel networks which are structurally distinct from controls, yet without substantially restricting or promoting tumor growth.

Tumors engineered to overexpress Ang ${ }^{*}$ are resistant to regression by VEGF blockade. We have previously reported that preformed vasculature in this model is largely ablated by treatment with VEGF Trap, with $80 \%$ regression of established tumors after 36 days of treatment (18). Biweekly injections of VEGF Trap ( $25 \mathrm{mg} / \mathrm{kg}$ intraperitoneally) were initiated. Control GFP-expressing control tumors were regressed by $80 \%$ at day $36(5.6 \pm 1.0 \mathrm{~g}$, day $0 \mathrm{vs} .1 .0 \pm 0.3 \mathrm{~g}$, day $36, \mathrm{P}=0.0003)$, reproducing the results observed in parental tumors (18). In contrast, Ang $1 *$-expressing tumors did not regress $(4.9 \pm 1.8 \mathrm{~g}$ at day 56 of treatment versus $4.5 \pm 1.1 \mathrm{~g}$ at day 0; Fig. 2), displayed no gross necrosis, and formed visibly increased surface vasculature by day 36 (data not shown). Growth of untreated Ang1*-expressing and GFP-expressing tumors was equivalent to previously described controls (18). We confirmed that tumor expression of Ang $1^{*}$ persisted at similar levels at day 0 and the end of the experiment (data not shown). Thus, tumor regression was prevented by Ang $1 *$ expression.

Ang $1^{*}$ expression reduces tumor hypoxia. If tumor perfusion had been stabilized by Ang1* during VEGF Trap treatment, oxygen delivery would be predicted to be maintained. We therefore assessed hypoxia using pimonidazole injection just prior to euthanizing mice. Whereas GFP-expressing tumors displayed widespread necrosis and hypoxia after 36 days of treatment, Ang1* tumors were much less necrotic and only minimally hypoxic (Fig. 3A). Reduction in hypoxia would be predicted to similarly influence expression of known hypoxia-regulated factors. Therefore, we examined expression of VEGF and CXCL12 in tumor extracts by real-time PCR. Whereas expression of both factors was significantly increased in VEGF Trap-treated control tumors, this effect was blunted in Ang1* expressing tumors, with a smaller increase in VEGF and no detectable increase in CXCL12 expression (Fig. 3B). Collectively, these results suggest that Ang1* expression reduced hypoxia, enabling tumors to persist.

Ang1* expression prevents vascular ablation by VEGF Trap. We have previously shown that VEGF inhibition causes rapid loss of vessels in the SK-NEP1 model, with ablation of small branches detected as early as $24 \mathrm{~h}$ after treatment (21). In contrast, VEGF Trap caused modest alterations in Ang1*expressing tumor vascular networks, which were most evident early in treatment. Pruning of small endothelial branches was detectable at days 1 and 5, although this effect was slight compared with the widespread vascular ablation noted at the same timepoints in our previous studies of SK-NEP1 (21). In order to analyze these changes quantitatively, we performed computerized image analysis of lectin perfusion studies (18) (Fig. 4A). Although the total number of vessels decreased by $51 \%$ between days 0 and 5 (Fig. 4B, vessels; $\mathrm{P}=0.005$ ), total vessel length and mean vascular density were largely maintained (Fig. 4B, MVD and length; both, $83 \%$ of day 0 values, $\mathrm{P}=\mathrm{NS}$ ). These changes are consistent with the relative preservation of larger vessels we observed histologically, with the early eradication of fine branches by VEGF Trap reflecting the importance of VEGF in maintaining this subset of vasculature. We confirmed the loss of small vascular branches by immunostaining for endothelial cells (PECAM-1) and mural cells ( $\alpha$ SMA), although by day 5 increased accumulation of mural cells was evident.

Administration of BowAng-1 prevents tumor regression by VEGF Trap. In order to confirm the ability of Tie-2 activation 


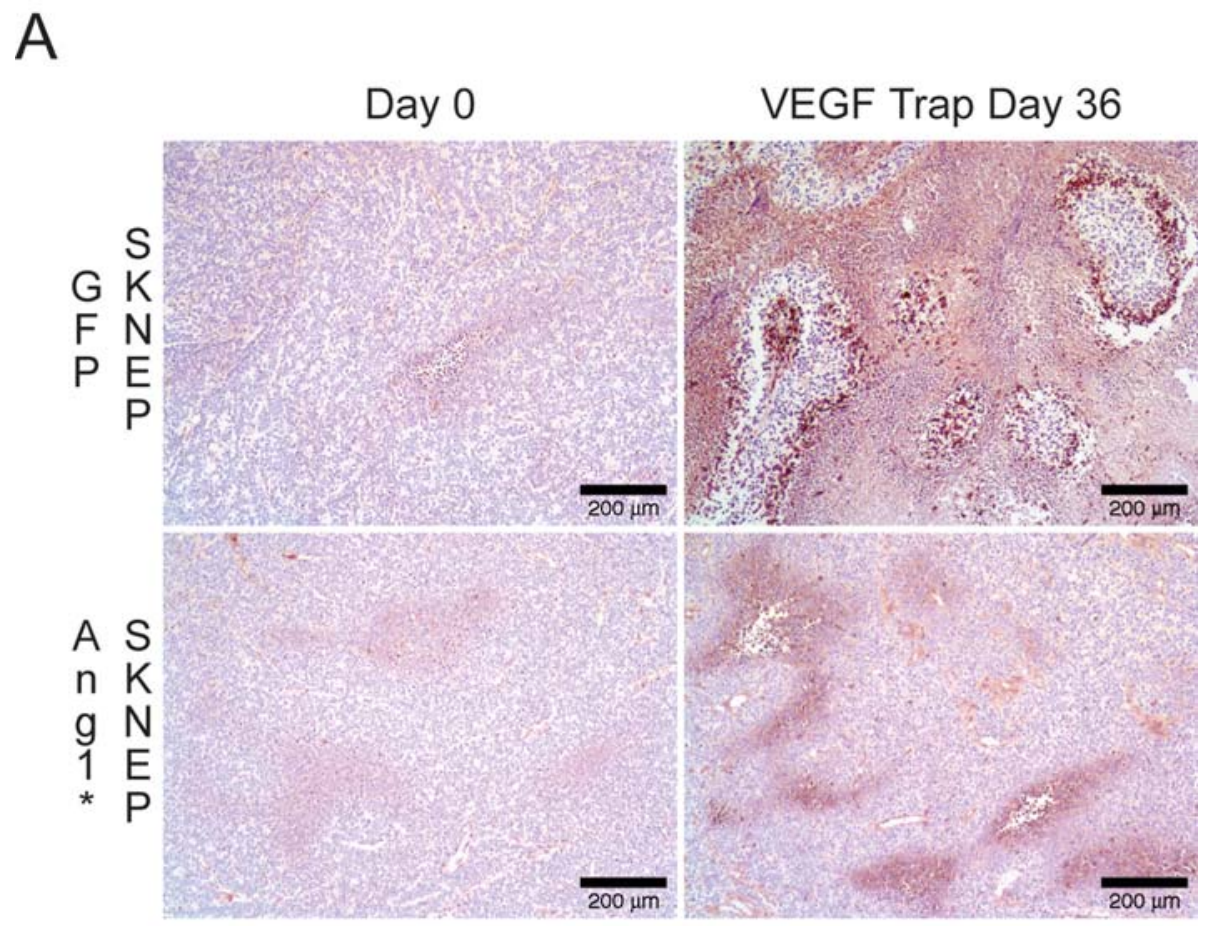

B
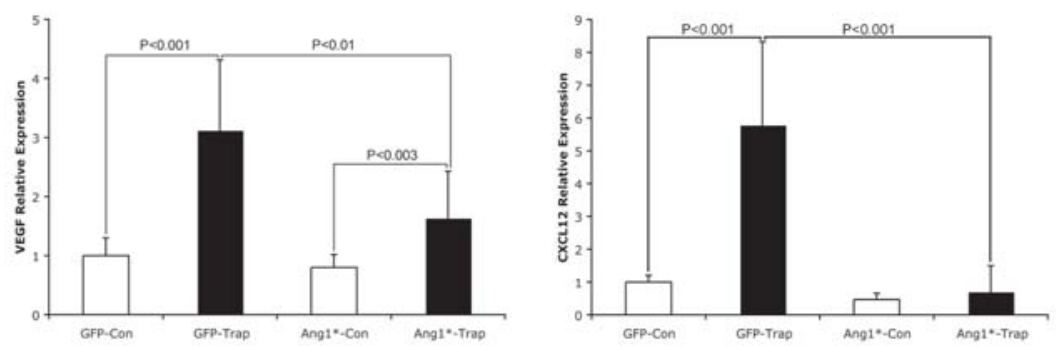

Figure 3. Tissue hypoxia is diminished in Ang1*-expressing tumors during VEGF Trap treatment. (A) Using pimonidazole staining, GFP-expressing controls were found to be extensively necrotic and hypoxic at day 36, whereas Ang1*-expressing xenografts were minimally hypoxic at the same time-point, consistent with comparatively robust perfusion. (B) VEGF Trap treatment stimulated expression of the hypoxia-regulated factors VEGF and CXCL12 (SDF-1) in GFPtransfected tumors, whereas expression was blunted in Ang1* expressing tumors, consistent with decreased hypoxia.

to protect tumors from regression induced by VEGF blockade, we tested the effect of administering an engineered angiopoietin tetramer which activates Tie-2 (Ang- $\mathrm{F}_{1}-\mathrm{Fc}_{-} \mathrm{F}_{1}$, BowAng-1 (19); Regeneron Pharmaceuticals; a schematic comparison of native Ang-1 with Ang1* and Bow-Ang-1 is shown in Fig. 5). SK-NEP1 tumor cells were implanted as above. After 29 days, mice with palpable tumors were randomly divided into three groups, receiving: i) BowAng-1 (25 mg/kg injected subcutaneously thrice weekly); ii) human IgG1 Fc protein $(25 \mathrm{mg} / \mathrm{kg}$ biweekly); and iii) no treatment. The last group was sacrificed 35 days after implantation [day (-7)] to establish baseline vessel architecture and tumor weight $(2.6 \pm 0.9 \mathrm{~g})$. After 13 days of pre-treatment (day 0), tumor weights in BowAng-1 and Fc pre-treatment groups were similar to each other $(4.0 \pm 1.3 \mathrm{~g}$ and $3.6 \pm 1.1 \mathrm{~g}$, respectively), consistent with our finding that Ang1* overexpression did not increase untreated tumor weights at day 0 (above).

Fc pre-treated mice were then randomly subdivided to receive: i) continued $\mathrm{Fc}$ injections; or ii) injections of VEGF Trap (25 mg/kg biweekly). BowAng-1 pre-treated mice remained on the same treatment regimen and additionally started to receive VEGF Trap injections $(25 \mathrm{mg} / \mathrm{kg}$ biweekly). Mice from each treatment group were euthanized on treatment days 5 or 20-27 for tumor weight evaluation. In the short term (day 5) VEGF Trap induced some tumor growth delay in both $\mathrm{Fc}$ and BowAng-1 pre-treated mice as compared to Fc-treated controls $(3.8 \pm 1.2 \mathrm{~g}$ and $4.2 \pm 2.2 \mathrm{~g}$ respectively, vs. $5.9 \pm 0.9$ g; Fig. 4). In the longer term, however, BowAng-1 strikingly decreased sensitivity of tumors to VEGF Trap, resulting in large tumors in BowAng-1 + VEGF Trap-treated mice comparable to Fc-treated controls $(7.8 \pm 1.9 \mathrm{~g}$ vs. $9.1 \pm 1.6 \mathrm{~g})$. Tumors in VEGF Trap-treated mice showed growth arrest or partial regression $(2.7 \pm 0.5 \mathrm{~g}, \mathrm{P}=0.043)$ consistent with our previous data (18). There was a linear correlation between circulating hVEGF/VEGF trap complex levels and renal tumor weights, indicating that VEGF Trap level and distribution seemed to be sufficient to capture all tumor-derived hVEGF. Taken together, these results indicate that sustained activation of Ang-1/Tie-2 is capable of sufficiently protecting tumors such that regression by VEGF 


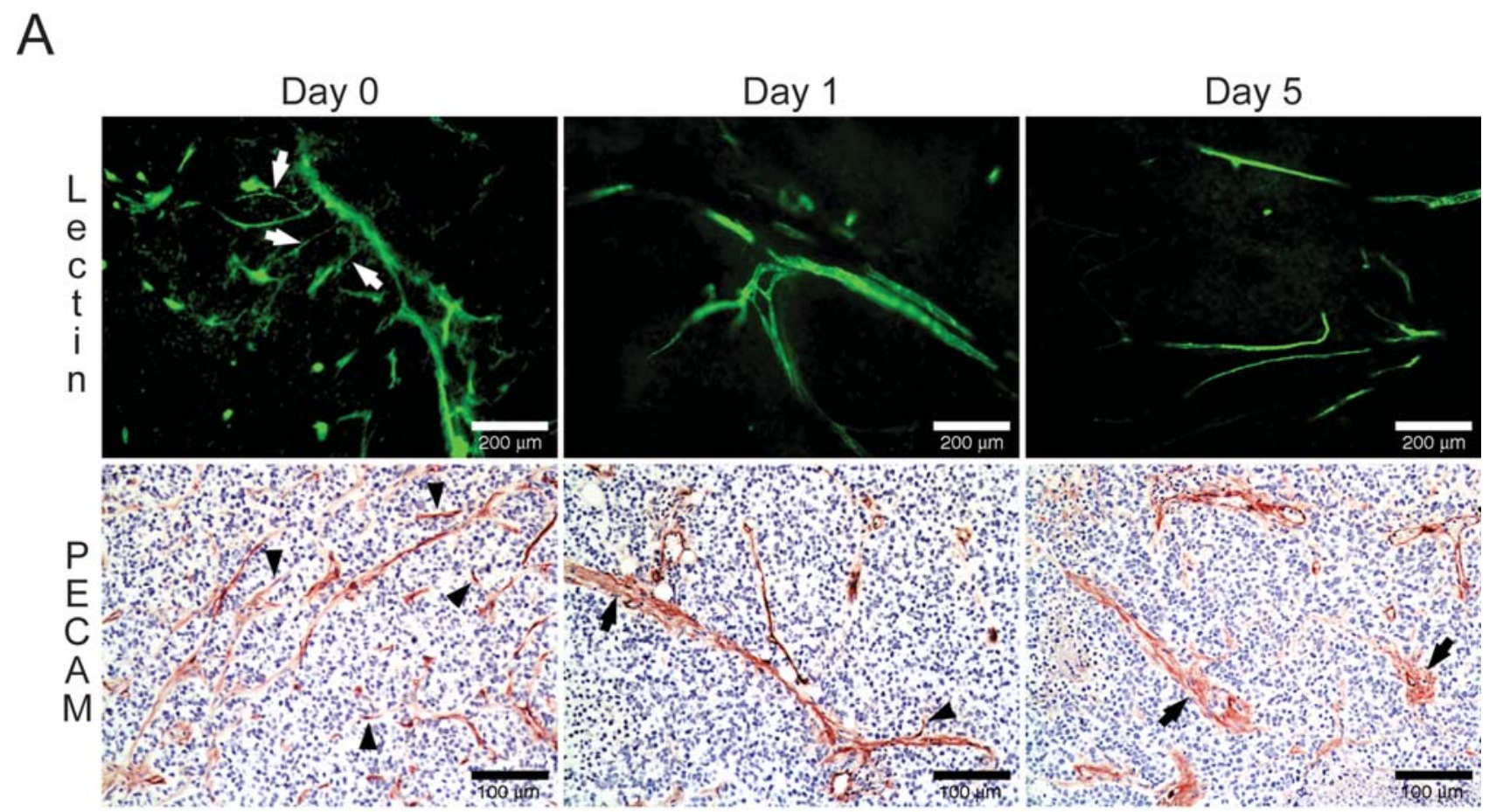

B
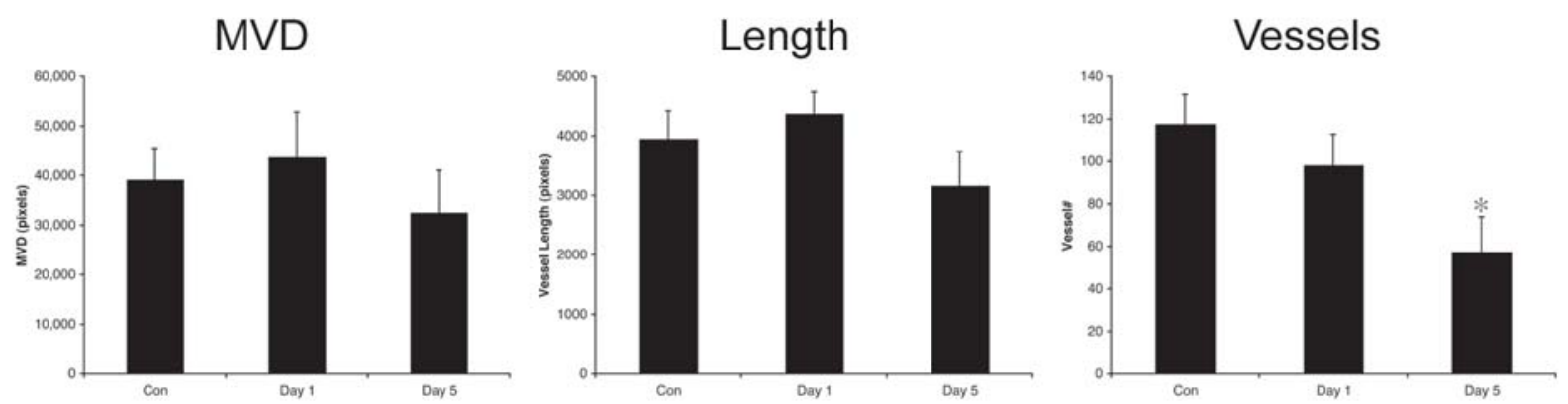

Figure 4. Remodeled vasculature in Ang1* tumors is resistant to destabilization by VEGF Trap. (A) L. esculentum perfusion studies demonstrate large vessels with an erratic network of fine branches in Ang1* tumors prior to treatment (day 0). These larger vessels persist despite ablation of the irregular small vessels after 1 and 5 days of VEGF Trap treatment, suggesting that this second population is VEGF-dependent. These alterations were strikingly different from those previously reported in perfusion studies of control SK-NEP1 xenografts during days 1 and 5 of VEGF Trap administration, in which the bulk of vasculature is eradicated by day 5 (21). Similar loss of fine branches was observed on PECAM immunostaining. (B) We quantitated these findings using computerized image analysis of lectin perfusion studies. Whereas vessel number decreased significantly by day 5 of treatment ( $51 \%$ of day 0 values; $\mathrm{P}=0.005)$, mean vessel density and vessel length were not significantly changed ( $83 \%$ of day 0 values; $\mathrm{P}=\mathrm{NS})$. Thus, vascular ablation is significantly blunted in Ang $1^{*}$-expressing tumors during VEGF Trap administration, as compared with SK-NEP-1 controls (18).

Trap is prevented, presumably by protecting vasculature and preserving tumor perfusion.

\section{Discussion}

Multiple groups have reported that inhibition of VEGF causes vascular remodeling, and can induce tumor expression of Ang-1. However, overexpression of Ang-1 stimulates divergent responses depending on tumor type, ranging from promotion to limitation of growth. These disparate observations suggest that Ang-1 may function in a context-dependent manner in neoplastic vascular beds. Tie- 2 activation plays a critical role in vessel integrity, both in developing embryos (23) and in adult vasculature, and can protect endothelial cells from microenvironmental stress by activating survival pathways $(24,25)$ and stimulating mural cell recruitment $(23,26)$. Overexpression of Ang-1 can restrict formation of new capillary sprouts, which appears to require temporary destabilization of the vascular wall by its partial antagonist Ang-2 in concert with VEGF (27-29). Thus, Ang-1 may be most likely to stabilize tumor perfusion and growth in systems with unstable or stressed vasculature, whereas it may limit growth in tumors dependent on rapid vascular sprout formation. Our SK-NEP1 model may represent an intermediate in this spectrum, since Ang 1* expression neither promoted nor limited tumor growth, although distinct architectural changes in vasculature were found. The increased diameter, mural cell recruitment, and reduction in fine branches in Ang1*-expressing tumors compared to SK-NEP1 controls are changes seen in vascular beds that survive when VEGF is withdrawn (4), and may be consistent with a shift to a vessel phenotype that is less dependent on VEGF signaling. 


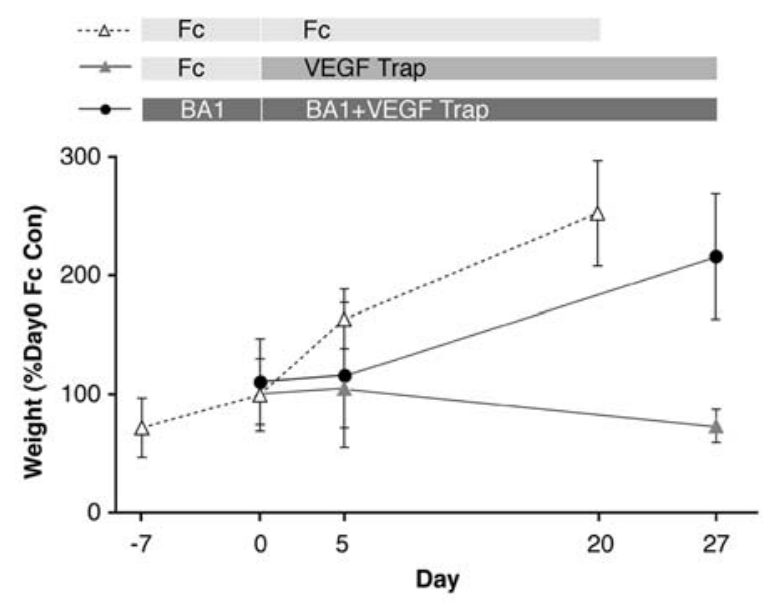

Figure 5. Injection of an Ang-1 agonist prevents regression and permits tumor growth during VEGF Trap treatment. In order to confirm the ability of Ang-1/Tie-2 signaling to protect tumors from regression induced by VEGF blockade, we injected an engineered angiopoietin tetramer which activates this axis [Ang- $\mathrm{F}_{1}-\mathrm{Fc}-\mathrm{F}_{1}$, BowAng-1 (19); Regeneron Pharmaceuticals]. SK-NEP-1 xenografts were allowed to grow for 29 days. Mice with palpable tumors were then randomly divided into three groups, receiving: i) injections of BowAng-1 (25 mg/kg delivered subcutaneously three times per week); ii) human IgG1 Fc protein ( $\mathrm{Fc} ; 25 \mathrm{mg} / \mathrm{kg}$ biweekly); and iii) no treatment. Untreated mice were sacrificed 35 days after implantation [day (-7)] to establish baseline vessel architecture and mean tumor weights $(2.6 \pm 0.9 \mathrm{~g})$ After 13 days of pre-treatment (day 0), tumor weights in both BowAng1 and Fc pre-treatment groups were similar to each other $(4.0 \pm 1.3 \mathrm{~g}$ and $3.6 \pm 1.1 \mathrm{~g}$, respectively). At this point, Fc pre-treated mice were randomly subdivided into two treatment groups that either continued to receive Fc (empty triangles) or Fc + VEGF Trap injections (filled triangles). BowAng1 pretreated mice remained on the same regimen and additionally started to receive VEGF Trap injections (filled circles). Mice were sacrificed on treatment day 5 or 27 for tumor weight evaluation $(\mathrm{N}=4$ for each treatment group at each time-point). At day 5, VEGF Trap caused xenograft growth delay in both Fc and BowAng1 pre-treated mice as compared to Fc treated controls $(3.8 \pm 1.2 \mathrm{~g}$ and $4.2 \pm 2.2 \mathrm{~g}$ respectively, vs. $5.9 \pm 0.9 \mathrm{~g})$. By day 27 , tumors in VEGF Trap treated mice showed partial regression (solid circles, $2.7 \pm 0.5 \mathrm{~g}, \mathrm{P}=0.043$, as compared to $\mathrm{Fc}$ treated mice, unfilled circles) consistent with our previous data (18). Strikingly, BowAng1 + VEGF Traptreated xenografts were comparable to the Fc-treated controls $(7.8 \pm 1.9 \mathrm{~g}$ vs $9.1 \pm 1.6 \mathrm{~g}$, solid triangles), indicating that sustained delivery of Ang-1 prevents regression by VEGF Trap.

Previous data support additional functions for Ang-1 that promote perfusion. Overexpression of Ang-1 in ischemic muscle increases maximal blood flow although resting flow is unchanged (30). Further, Ang-1 can stimulate the assembly of higher-order vessel networks, restoring hierarchy to retinal vessels even when pericytes are absent (12). Taken together, these data suggest that Tie- 2 signaling can acutely contribute to the appropriate distribution of blood supply in response to tissue demand. In addition, recent work demonstrates that Ang-1 can cause endothelial proliferation leading to enlargement of pre-existing vessels (31). Thus, these properties may have contributed to maintenance of the blood supply during VEGF blockade in tumors overexpressing Ang1* or treated with BowAng-1.

In contrast to the virtual ubiquity of VEGF in human cancers, responses to VEGF blockade differ between tumor types. Similarly, expression of Ang-1 in clinical samples is variable. The role of Ang-1 in tumor angiogenesis has consequently been controversial. In our model, overexpression of Ang 1* and administration of BowAng-1 profoundly altered the response to VEGF blockade. Whereas control and GFP-expressing tumors were initially $80 \%$ regressed by VEGF Trap, Ang1*-expressing xenografts were not regressed, and BowAng1-treated tumors actually grew. Our findings suggest that Tie-2 activation may most strongly influence tumor growth when VEGF signaling has been suppressed in a VEGF-dependent system, as a modulator of the response to sustained hypoperfusion. It is not yet known how generally this phenomenon will apply to other tumor types or sites of tumor growth. We have investigated the effects of Ang1* on heterotopic subcutaneous tumor growth using SK-NEP1 cells and C6 glioma cells coimplanted with Matrigel, and have not seen vascular resistance to the effects of VEGF inhibition on tumor growth. While we have focused in the current study on possible vascular changes mediated by Tie- 2 activation as a means of tumor resistance, the precise mechanisms by which this axis promotes the apparent resistance to VEGF blockade in orthotopic SK-NEP1 tumors remain to be completely elucidated.

Thus, these studies support a model in which Ang-1/Tie-2 signaling can shift vasculature to a less VEGF-dependent state, functioning as a compensatory mechanism when tumor vasculature is stressed by VEGF blockade. In the postnatal trachea, Ang-1 can protect vasculature from regression by VEGF Trap (32), indicating that it can protect preformed vessels from VEGF withdrawal in the physiologic setting. Our findings extend these data by providing evidence that Ang-1/Tie-2 activation can remodel pathologic vasculature prior to VEGF blockade and rescue perfusion when treatment begins. Together these effects are likely to decrease VEGF dependence of tumor vasculature, maintaining the blood supply and survival of neoplastic cells.

Targeting vasculature has been validated as a cancer therapy. The specific success of VEGF blockade has propelled agents that block this molecule into widely used clinical protocols. It is equally clear that this therapy varies in effectiveness between tumor types, and that even patients with meaningful responses may ultimately experience tumor progression. Our results provide evidence that Tie-2 activation can protect tumor vasculature from destabilization by VEGF blocking agents, and suggest that investigating this pathway as a potential contributor to vessel survival during chronic microenvironmental stress may be a rational next step.

\section{Acknowledgments}

The authors thank Ms. Lucy Eljuga for technical assistance. These studies were supported by grants from the Pediatric Cancer Foundation (J.J.K. and D.J.Y.), tay-bandz, Inc. (D.J.Y.), the Sorkin Fund (JJK), and National Cancer Institute grants RO1-CA088951 (D.J.Y.), R01-CA100451 (J.J.K.), and K08CA107077 (J.H.).

\section{References}

1. Hurwitz H, Fehrenbacher L, Novotny W, Cartwright T, Hainsworth J, Heim W, Berlin J, Baron A, Griffing S, Holmgren E, Ferrara N, Fyfe G, Rogers B, Ross R and Kabbinavar F: Bevacizumab plus irinotecan, fluorouracil, and leucovorin for metastatic colorectal cancer. N Engl J Med 350: 2335-2342, 2004. 
2. Yang JC, Haworth L, Sherry RM, Hwu P, Schwartzentruber DJ Topalian SL, Steinberg SM, Chen HX and Rosenberg SA: A randomized trial of bevacizumab, an anti-vascular endothelial growth factor antibody, for metastatic renal cancer. N Engl J Med 349: 427-434, 2003

3. Benjamin LE, Hemo I and Keshet E: A plasticity window for blood vessel remodelling is defined by pericyte coverage of the preformed endothelial network and is regulated by PDGF-B and VEGF. Development 125: 1591-1598, 1998.

4. Benjamin LE, Golijanin D, Itin A, Pode D and Keshet E: Selective ablation of immature blood vessels in established human tumors follows vascular endothelial growth factor withdrawal. J Clin Invest 103: 159-165, 1999.

5. Huang J, Soffer SZ, Kim ES, McCrudden KW, Huang J, New T, Manley CA, Middlesworth W, O'Toole K, Yamashiro DJ and Kandel JJ: Vascular remodeling marks tumors that recur during chronic suppression of angiogenesis. Mol Cancer Res 2: $36-42,2004$.

6. Erber R, Thurnher A, Katsen AD, Groth G, Kerger H, Hammes HP, Menger MD, Ullrich A and Vajkoczy P: Combined inhibition of VEGF and PDGF signaling enforces tumor vessel regression by interfering with pericyte-mediated endothelial cell survival mechanisms. FASEB J 18: 338-340, 2004.

7. Casanovas O, Hicklin DJ, Bergers G and Hanahan D: Drug resistance by evasion of antiangiogenic targeting of VEGF signaling in late-stage pancreatic islet tumors. Cancer Cell 8: 299-309, 2005

8. Gerber HP, Kowalski J, Sherman D, Eberhard DA and Ferrara N: Complete inhibition of rhabdomyosarcoma xenograft growth and neovascularization requires blockade of both tumor and host vascular endothelial growth factor. Cancer Res 60: 6253-6258, 2000

9. Stoeltzing O, Ahmad SA, Liu W, McCarty MF, Wey JS, Parikh AA, Fan F, Reinmuth N, Kawaguchi M, Bucana CD and Ellis LM: Angiopoietin-1 inhibits vascular permeability, angiogenesis, and growth of hepatic colon cancer tumors Cancer Res 63: 3370-3377, 2003

10. Hawighorst T, Skobe M, Streit M, Hong YK, Velasco P Brown LF, Riccardi L, Lange-Asschenfeldt B and Detmar M: Activation of the tie 2 receptor by angiopoietin-1 enhances tumor vessel maturation and impairs squamous cell carcinoma growth. Am J Pathol 160: 1381-1392, 2002.

11. Machein MR, Knedla A, Knoth R, Wagner S, Neuschl E and Plate KH: Angiopoietin-1 promotes tumor angiogenesis in a rat glioma model. Am J Pathol 165: 1557-1570, 2004.

12. Uemura A, Ogawa M, Hirashima M, Fujiwara T, Koyama S, Takagi H, Honda Y, Wiegand SJ, Yancopoulos GD and Nishikawa S: Recombinant angiopoietin-1 restores higher-order architecture of growing blood vessels in mice in the absence of mural cells. J Clin Invest 110: 1619-1628, 2002

13. Baluk P, Lee CG, Link H, Ator E, Haskell A, Elias JA and McDonald DM: Regulated angiogenesis and vascular regression in mice overexpressing vascular endothelial growth factor in airways. Am J Pathol 165: 1071-1085, 2004.

14. Zhou YF, Stabile E, Walker J, Shou M, Baffour R, Yu Z, Rott D, Yancopoulos GD, Rudge JS and Epstein SE: Effects of gene delivery on collateral development in chronic hypoperfusion: diverse effects of angiopoietin-1 versus vascular endothelial growth factor. J Am Coll Cardiol 44: 897-903, 2004.

15. Sandhu R, Teichert-Kuliszewska K, Nag S, Proteau G, Robb MJ, Campbell AI, Kuliszewski MA, Kutryk MJ and Stewart DJ: Reciprocal regulation of angiopoietin-1 and angiopoietin-2 following myocardial infarction in the rat. Cardiovasc Res 64 $115-124,2004$

16. Winkler F, Kozin SV, Tong RT, Chae SS, Booth MF, Garkavtsev I, Xu L, Hicklin DJ, Fukumura D, Di Tomaso E, Munn LL and Jain RK: Kinetics of vascular normalization by VEGFR2 blockade governs brain tumor response to radiation: role of oxygenation, angiopoietin-1, and matrix metalloproteinases. Cancer Cell 6: 553-563, 2004

17. Findley CM, Cudmore MJ, Ahmed A and Kontos CD: VEGF induces Tie2 shedding via a phosphoinositide 3-kinase/Akt dependent pathway to modulate Tie2 signaling. Arterioscler Thromb Vasc Biol 2: 2619-2626, 2007.
18. Huang J, Frischer JS, Serur A, Kadenhe A, Yokoi A, McCrudden KW, New T, O'Toole K, Zabski S, Rudge JS, Holash J, Yancopoulos GD, Yamashiro DJ and Kandel JJ: Regression of established tumors and metastases by potent vascular endothelial growth factor blockade. Proc Natl Acad Sci USA 100: 7785-7790, 2003

19. Davis S, Papadopoulos N, Aldrich TH, Maisonpierre PC, Huang T, Kovac L, Xu A, Leidich R, Radziejewska E, Rafique A, Goldberg J, Jain V, Bailey K, Karow M, Fandl J, Samuelsson SJ, Ioffe E, Rudge JS, Daly TJ, Radziejewski C and Yancopoulos GD: Angiopoietins have distinct modular domains essential for receptor binding, dimerization and superclustering. Nat Struct Biol 10: 38-44, 2003.

20. Maisonpierre PC, Suri C, Jones PF, Bartunkova S, Wiegand SJ, Radziejewski C, Compton D, McClain J, Aldrich TH, Papadopoulos N, Daly TJ, Davis S, Sato TN and Yancopoulos GD: Angiopoietin-2, a natural antagonist for Tie2 that disrupts in vivo angiogenesis. Science 277: 55-60, 1997.

21. Huang J, Frischer JS, New T, Kim ES, Serur A, Lee A, Kadenhe-Chiwishe A, Pollyea DA, Yokoi A, Holash J, Yancopoulos GD, Kandel JJ and Yamashiro DJ: TNP-470 promotes initial vascular sprouting in xenograft tumors. Mol Cancer Ther 3: 335-343, 2004.

22. Thurston G, Suri C, Smith K, McClain J, Sato TN, Yancopoulos GD and McDonald DM: Leakage-resistant blood vessels in mice transgenically overexpressing angiopoietin-1. Science 286: 2511-2514, 1999.

23. Suri C, Jones PF, Patan S, Bartunkova S, Maisonpierre PC, Davis S, Sato TN and Yancopoulos GD: Requisite role of angiopoietin-1, a ligand for the TIE2 receptor, during embryonic angiogenesis. Cell 87: 1171-1180, 1996.

24. Kim I, Kim HG, So JN, Kim JH, Kwak HJ and Koh GY: Angiopoietin-1 regulates endothelial cell survival through the phosphatidylinositol 3'-Kinase/Akt signal transduction pathway. Circ Res 86: 24-29, 2000.

25. Daly C, Wong V, Burova E, Wei Y, Zabski S, Griffiths J, Lai KM, Lin HC, Ioffe E, Yancopoulos GD and Rudge JS: Angiopoietin-1 modulates endothelial cell function and gene expression via the transcription factor FKHR (FOXO1). Genes Dev 18: 1060-1071, 2004.

26. Iivanainen E, Nelimarkka L, Elenius V, Heikkinen SM, Junttila TT, Sihombing L, Sundvall M, Maatta JA, Laine VJ, Yla-Herttuala S, Higashiyama S, Alitalo K and Elenius K: Angiopoietin-regulated recruitment of vascular smooth muscle cells by endothelial-derived heparin binding EGF-like growth factor. FASEB J 17: 1609-1621, 2003.

27. Holash J, Wiegand SJ and Yancopoulos GD: New model of tumor angiogenesis: dynamic balance between vessel regression and growth mediated by angiopoietins and VEGF. Oncogene 18: 5356-5362, 1999

28. Holash J, Maisonpierre PC, Compton D, Boland CR, Alexander D, Yancopoulos GD and Weigand SJ: Vessel cooption, regression, and growth in tumors mediated by angiopoietins and VEGF. Science 284: 1994-1998, 1999.

29. Yancopoulos GD, Davis S, Gale NW, Rudge JS, Wiegand SJ and Holash J: Vascular-specific growth factors and blood vessel formation. Nature 407: 242-248, 2000.

30. Shyu KG, Manor O, Magner M, Yancopoulos GD and Isner JM: Direct intramuscular injection of plasmid DNA encoding angiopoietin-1 but not angiopoietin-2 augments revascularization in the rabbit ischemic hindlimb. Circulation 98: 2081-2087, 1998.

31. Thurston G, Wang Q, Baffert F, Rudge J, Papadopoulos N, Jean-Guillaume D, Wiegand S, Yancopoulos GD and McDonald DM: Angiopoietin 1 causes vessel enlargement, without angiogenic sprouting, during a critical developmental period. Development 132: 3317-3326, 2005.

32. Baffert F, Thurston G, Rochon-Duck M, Le T, Brekken R and McDonald DM: Age-related changes in vascular endothelial growth factor dependency and angiopoietin-1-induced plasticity of adult blood vessels. Circ Res 94: 984-992, 2004. 